VOL. $2(1970), 280$.

\title{
Some characterisations of the ellipsoid and the Minkowski theory of reduction
}

\author{
P. W. Aitchison
}

The Minkowski theory of reduction of $n$-ary, positive definite quadratic forms is concerned with properties of certain such forms, called reduced forms, especially when the variables take integer values. Each such form may represent an ellipsoid in $n$-dimensional Euclidean space, $R^{n}$, and then the theory may be formulated geometrically in terms of the relations between a fixed ellipsoid and a variable lattice. The theory of reduction of a convex body $K$ is the natural generalisation of the Minkowski reduction theory, obtained by replacing the ellipsoid by $K$.

Minkowski obtained, among many other results, two finiteness theorems, namely: finitely many linear inequalities in the coefficients are sufficient to determine all reduced forms; only finitely many unimodular transformations can transform a reduced form again into a reduced form. It is natural to ask whether the geometrical version of these results can be generalised, and in fact Minkowski showed that both hold in $R^{2}$ for convex bodies other than the ellipsoid.

This thesis shows that in $R^{n}$, for $n \geq 3$, neither of the results hold for a convex body $K$ other than the ellipsoid. The proof involves a new characterisation of the ellipsoid, namely: if any two parallel planes "sufficiently close" to a tac plane of $K$, intersect $K$ in equivalent convex bodies, then $K$ is an ellipsoid. Here, two convex bodies are equivalent if, for some Minkowski metric, both have constant width. For example, two bodies of constant width are equivalent, or two homothetic bodies.

Received 2 April 1969. Thesis submitted to the Australian National University, October 1968. Degree approved, July 1969. Supervisors: Professor K. Mahler, Dr. V. Dlab. 